\title{
Mikrofluidisches Microarray für die Einzelzellanalyse
}

\author{
M. Rothbauer, P. Schuller, R. Afkhami, H.D. Wanzenboeck, P. Ertl, H. Zirath
}

\begin{abstract}
In diesem Artikel stellen wir die Entwicklung eines mikrofluidischen Microarray zum Immobilisieren und Analysieren einzelner Zellen vor. Das System besteht aus einem Hybridchip aus Polydimethylsiloxan und Glas mit einem mikrofluidischen Kanalnetz, welches mit hydrodynamischen Fallen ausgestattet ist. Der Mikrofluidik-Chip wurde mit MEMS-Technologie hergestellt und anschließend durch experimentelle Strömungsvisualisierung mit fluoreszierenden Partikeln charakterisiert. Die Fähigkeit zum Einfangen einzelner Zellen und die Performance des Systems werden mit zwei verschiedenen Zelltypen demonstriert und die Leistung für toxikologische Studien bewertet. Das entwickelte System konnte erfolgreich zum Einfangen, Aufrechterhalten und zum toxikologischen Screening einzelner Zellen eingesetzt werden, wobei ein Experiment nur wenige Stunden $(<3 \mathrm{~h})$ in Anspruch nimmt.
\end{abstract}

Schlüsselwörter: Mikrofluidik; MEMS; Photolithographie; Softlithographie; Einzelzellen; Toxikologie

\section{Microfluidic microarray for single-cell analysis.}

In this paper we present the development of a microfluidic microarray for trapping and analysis of single cells. The system consists of a PDMS/glass chip with a microfluidic channel network and hydrodynamic traps. The microfluidic chip was fabricated with MEMS technology and thereafter characterized by experimental flow visualization using fluorescent particles. Cell-capturing ability and overall performance of the system are demonstrated by employing two different cell types. Its performance for toxicological studies are evaluated. The developed microfluidic system successfully trapped and maintained mammalian cells under hydrodynamic conditions. Practical application involved single-cell toxicology screening resulting in a total assay time of less than three hours.

Keywords: microfluidics; MEMS; photolithography; soft lithography; single cell; toxicology

Eingegangen am 31. Jänner 2020, angenommen am 28. Februar 2020, online publiziert am 16. März 2020 (C) The Author(s) 2020

\section{Einleitung}

Mikrofluidiktechnologie, die Flüssigkeiten in Kanälen mit Abmessungen im Mikrometerbereich manipulieren kann, bietet Forschern und der Industrie seit Anfang der 1980er Jahre neue und vielversprechende Werkzeuge für eine Vielzahl von Anwendungen. Bei Herstellungsverfahren, die aus der Siliziummikroelektronik und aus mikroelektromechanischen Systemen (MEMS) stammen, bestanden in der frühen Ära der Mikrofluidik die Bauelemente aus Glas oder Silizium mit durch Photolithographie hergestellten Strukturen [24]. Seit den späten Achtzigern wurde diese MEMS-basierte Technologie vermehrt auch von Biologen für zelluläre Studien genutzt, angefangen mit der Kultivierung von Zellen auf Mikromustern, um den Effekt der Mikroarchitektur auf das Zellwachstum zu untersuchen [9]. Nach der Entwicklung der Soft-Lithographie mit Polydimethylsiloxan (PDMS) als Verfahren zum Formen von mikrofabrizierten Strukturen wurden die Vielseitigkeit und die Kosten von mikrofluidischen Vorrichtungen stark verringert, wodurch sich die Verfügbarkeit erhöhte. Dieser Technologiesprung machte die Mikrofluidik zu einem wirklich leistungsstarken Instrument für die chemische Analyse und für biologische Studien. Insbesondere die bioverträglichen Eigenschaften des Polymers wie Sauerstoffdurchlässigkeit und Funktionalisierungsmöglichkeit haben zu einer raschen Weiterentwicklung der Technologie geführt $[6,14,26]$. Parallel dazu wurde durch Miniaturisierung und Integration von Sensoren, Ventilen und Aktuatoren in die Geräte die Entwicklung von Lab-on-a-Chip-Systemen (LOC) oder Mikrototalanalysesystemen ( $\mu$ TAS) eingeleitet $[5,24]$. Mit der Einführung von Mikrofluidik in die Zellkultur wurde es möglich, kleine Mengen an Flüssigkeit für die Zellmanipulation zu handhaben und abzugeben. Dadurch konnten entscheidende Einschränkungen der Standardformate für Zell-basierte Bioassays wie z.B. Temperatur- und Gaskontrolle, genaue Kontrolle über Geometrie, Nährstoffversorgung und Scherbelastung, Abfallbeseitigung, chemische Verabreichung, Assay-Parallelisierung sowie Zellkulturautomatisierung überwunden werden. Die ersten in vitro Modelle basierten auf einem Zelltyp, aber die Erkenntnis, dass die Mikrofabrikation die präzise Manipulation von Zell-Zell-Wechselwirkungen ermöglicht, führte zur Entwicklung fortschrittlicher Geräte für das Tissue Engineering, so genannter Organ-on-a-Chip-Systeme [1, 19, 21, 25]. Die Mehrzahl der zellulären Studien wird an Massenzellverbänden von hunderttausenden Zellen durchgeführt, jedoch treten innerhalb derselben Zellpopulation bereits innerhalb eines Kulturgefäßes Unterschiede in Phänotyp und Genotyp auf, die als zelluläre Heterogenität bezeichnet werden. Um Krankheiten wie Krebs, Schlaganfall und Herzerkrankungen zu untersuchen, ist es wichtig, auch die Heterogeni-

Rothbauer, Mario, Institut für Angewandte Synthesechemie, Technische Universität Wien, Getreidemarkt 9/E163, 1060 Wien, Österreich; Schuller, Patrick, Institut für Festkörperelektronik, Technische Universität Wien, Gußhausstraße 25-25a/E362, 1040 Wien, Österreich; Afkhami, Reza, Institut für Angewandte Synthesechemie, Technische Universität Wien, Getreidemarkt 9/E163, 1060 Wien, Österreich; Wanzenboeck, Heinz D., Institut für Festkörperelektronik, Technische Universität Wien, Gußhausstraße 25-25a/E362, 1040 Wien, Österreich; Ertl, Peter, Institut für Angewandte Synthesechemie, Technische Universität Wien, Getreidemarkt 9/E163, 1060 Wien, Österreich; Zirath, Helene, Institut für Angewandte Synthesechemie, Technische Universität Wien, Getreidemarkt 9/E163, 1060 Wien, Österreich (E-Mail: helene.zirath@tuwien.ac.at) 
tät des zellulären Lebenszyklus zu verstehen [11, 15]. In ähnlichen Studien zur Toxikologie wurde über Heterogenität bei der Aufnahme und der zellulären Reaktion berichtet, was darauf hinweist, dass neben der Massenanalyse auch eine Einzelzellanalyse durchgeführt werden muss [13, 22]. Die MEMS-Technologie bietet eine einzigartige Möglichkeit, Mikrostrukturen in Zellgröße zu entwerfen und herzustellen, um Zellpopulationen auf Einzelzellenebene zu zerlegen und zu isolieren. Das Einfangen von Zellen in der Mikrofluidik wurde mit verschiedenen Ansätzen durchgeführt, beispielsweise mit Mikrotiterplatten [18], Dielektrophorese [17], akustische Oberflächenwellen [8], Einkapselung in Mikrotröpfchen [10] und hydrodynamisches Einfangen $[3,20]$. Die am häufigsten verwendete Methode zum Einfangen einzelner Zellen wurde mit hydrodynamischem Fluss mit verschiedenen Designs durchgeführt [7]. Im Wesentlichen werden Zellen durch Fokussierung des Flusses vom Haupttransportkanal in Seitenkanäle oder hydrodynamische Fallen gesaugt und dadurch zur weiteren Analyse auf Einzelzellenebene immobilisiert. In einer frühen Arbeit von Valero et al. wurden Fangstellen für lebende Zellen geschaffen, indem zwei Kanäle mit Mikrolöchern verbunden wurden, in denen Zellen eingefangen und auf apoptotische Zelltoddynamik analysiert wurden (5 Zellen pro Gerät) [23]. BeneventeBabace et al. entwickelten eine Plattform mit einem $500 \mu \mathrm{m}$ breiten Kanal mit siebartiger Strukturen zum Einfangen von Zellen [2]. Durch Anlegen eines Gleichstroms von einem zweiten Einlass (CoFlow) könnten Zellen in die Fallen geleitet werden. In einem Ansatz mit einer höheren Zellzahl berichteten Chen et al. über eine Plattform, die Hunderte bis Tausende von Zellen für klonale Analysen einfangen kann, indem U-förmige hydrodynamische Fallen in eine stromabwärtige Wand von Mikrovertiefungen eingebaut werden [4]. Das Wiederauffinden eingefangener Einzelzellen als wichtige Überlegung für die weitere biologische Analyse kann mit diesem Mikrofluidsystem jedoch nicht realisiert werden. In der Arbeit von Sauzade et al. wird eine Lösung zum Wiederauffinden von Zellen nach dem Einfangen in hydrodynamischen Fallen vorgestellt [20]. In einem Strömungskanal mit mäanderförmiger Form verkürzen Einfangwege die Kanäle vor jeder Kurve über einen verengten Einfangkanal. Eine wellenförmige Struktur innerhalb der Kanäle führt ankommende Zellen zum Einfangweg und somit werden Zellen erfasst. Nach dem Einfangen wird Öl durch das System injiziert, wodurch die Zellen in Tröpfchen eingekapselt werden. Durch Umkehren des Ölflusses können die eingekapselten Zellen zur Analyse gewonnen werden.

In diesem Artikel stellen wir eine neuartige Kanalstruktur mit hydrodynamischen Fallen vor, bei der einzelne Zellen in Taschen eingefangen werden, ohne dass Führungsstrukturen oder laminarer Co-Flow erforderlich sind. Nach dem Einfangen der Zellen können Zellanalysen direkt am Chip durchgeführt werden sowie zur weiteren Analyse nach Abschluss des Einzelzell-Assays wiedergewonnen werden. Jede Zelle ist über enge Kanäle mit Zellkulturmedium verbunden und ermöglicht dadurch eine identische Versorgung aller Zellen. Die mikrofluidische Kanalstruktur besteht aus einem $50 \mu \mathrm{m}$ breiten Einlasskanal, der in zwei $30 \mu \mathrm{m}$ breite parallele Mäanderkanäle unterteilt ist, in denen jede Mäanderecke eine hydrodynamische Falle enthält, die so dimensioniert ist, dass sie eine einzelne Zelle einfangen und zurückhalten kann (Abb.1). Jede Zellfalle umschließt einen. Auslass, der mit einem separaten Kanal verbunden ist, der alle Auslässe miteinander verbindet und schließlich mit dem Auslass des mikrofluidischen Mikroarrays verbunden wird. Während der Perfusion der beiden Hauptkanäle teilt sich der Fluss in jeder Kurve in zwei Ströme, von denen einer dem Hauptkanal folgt und der Zweite durch die Zellenerfassungsbereiche in den Seitenkanal eintritt. Wenn eine Zelle durch den Hauptkanal transportiert

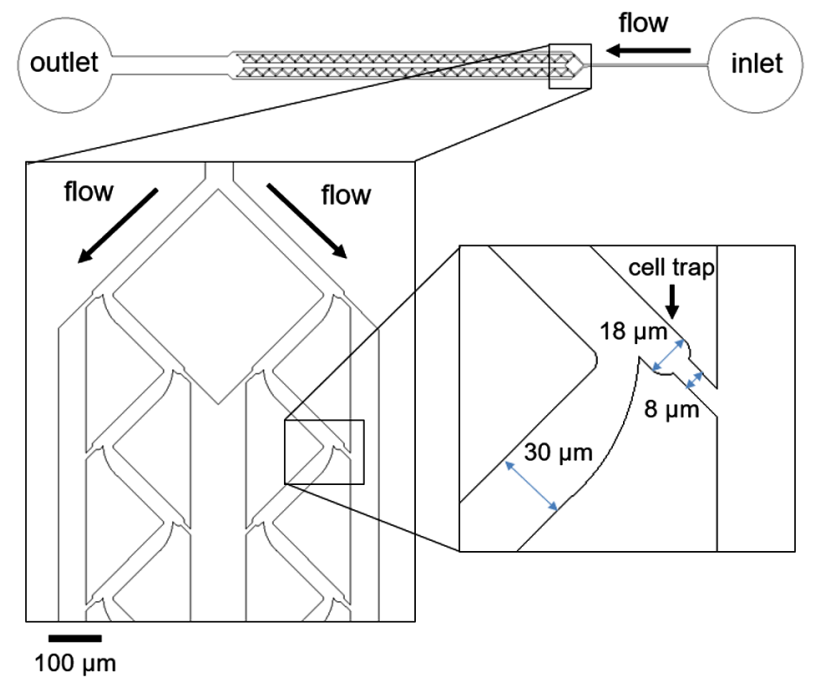

Abb. 1. Oben: Draufsicht auf das Einzelzellen-Chip-Design; unten links: Zoom in den Startbereich der Trap-Design, unten rechts: Zoom in eine einzelne Zellen-Trap mit Trap-Dimensionen

wird, tritt sie in den Hohlraum ein, wird hydrodynamisch immobilisiert und unterbricht dadurch den Fluss durch die Zellfalle, wodurch der Fluss nur im Hauptkanal fortgesetzt wird. Die nächste ankommende Zelle, die durch den Hauptkanal transportiert wird, passiert ohne Immobilisierung diese erste besetzte Falle. Dieses passive Einfangen von Zellen wird fortgesetzt, bis alle Hohlräume besetzt sind. Alle mikrofluidischen Strukturen haben eine Höhe von $25 \mu \mathrm{m}$ und jeder Chip besteht aus insgesamt 102 Zellenfallen. Der Chip wurde mit MEMS-Technologie hergestellt und durch experimentelle Strömungs-visualisierungsversuche mit fluoreszierenden Partikeln charakterisiert. Das Einfangen von Zellen wurde sowohl mit epithelialen als auch mit mesenchymalen Stammzellen getestet, und anschließend das Zellverhalten in den Einfanghohlräumen bewertet. Schließlich wurden zytotoxische Mittel, einschließlich Chlorpromazin getestet und die Lebensfähigkeit der Zellen unter Verwendung eines Standard-Farbstoff-ausschlusstests auf der Basis von Ethidiumbromid und Höchst-Nuclein-Färbung bewertet.

\section{Geräteherstellung}

Zur Herstellung des Mikrofluidik-Microarray-Chips wurden StandardMEMS-Technologie und weiche Lithografie Techniken angewendet. Für die Masterform wurden ein 4-Zoll-Silizium (Si) -Wafer und ein auf Epoxid basierender Fotolack (SU-8 2015, Microchem, US) verwendet. Die Form wurde unter Verwendung des folgenden photolithographischen Prozesses hergestellt. Vor dem Aufbringen des Photoresists wurde das Siliziumsubstrat mit einer Piranha-Nassätzung $\left(\mathrm{H}_{2} \mathrm{SO}_{4} / \mathrm{H}_{2} \mathrm{O}_{2}\right)$ gereinigt und anschließend mit $d \mathrm{dH}_{2} \mathrm{O}$ und Isopropanol gespült. Eine $25 \mu \mathrm{m}$ hohe Resistschicht wurde mit einem Schleuder-Beschichter (Polos SPIN150i) aufgebracht und danach vier min bei $95^{\circ} \mathrm{C}$ weichgebrannt. Nach dem weichen Einbrennen wurden die Proben mit einem Maskenausrichter (Suss MJB3 UV300) und einer im eigenen Haus hergestellten Borosilikatglas Maske (DWL66 Laserwriter, Heidelberg Instruments Mikrotechnik $\mathrm{GmbH}$, Deutschland) UV-Licht ausgesetzt. Zur thermischen Vernetzung des Harzes wurde ein Nachbelichtungsbrennen (PEB) bei $95^{\circ} \mathrm{C}$ für fünf min durchgeführt. Danach wurden die Proben in mr-Dev 600 (Micro Resist Technology $\mathrm{GmbH}$, Deutschland) bei Raumtemperatur entwickelt, bis sich alle unbelichteten Teile des Photoresists aufgelöst hatten. Nach der Entwicklung wurden die Proben in Isopropanol 
gespült und mit einer $\mathrm{N}_{2}$-Sprühpistole geblasen. Die Photoresiststrukturen wurden $30 \mathrm{~min}$ lang bei $150^{\circ} \mathrm{C}$ hartgebrannt. Vor der Verwendung wurde die Oberfläche der Form mit einer Antihaftbeschichtung bedeckt, um die Struktur während der weichen Lithografie zu schützen. Die Beschichtung wurde aufgebracht, indem die Formen mit $4 \mu \mathrm{L}$ Silan $(1 \mathrm{H}, 1 \mathrm{H}, 2 \mathrm{H}, 2 \mathrm{H}$-Perfluor-octyltriethoxysilan, Sigma-Aldrich) fünf min lang unter Vakuum gesetzt wurden und danach die Formen eine Stunde lang bei $70^{\circ} \mathrm{C}$ gebrannt wurden, um das zugängliche Silan zu verdampfen. Die Herstellung des PDMSMikrofluidik-Chips erfolgte durch Mischen von Sylgard 184 und Härtungsmittel (Dow Corning) im Verhältnis 10:1, Zentrifugieren bei 3200 RPM für fünf Minuten und Gießen über die gereinigte Silizium-Masterform, um eine fünf $\mathrm{mm}$ dicke PDMS-Schicht zu bilden. Nach einstündigem Härten bei $70^{\circ} \mathrm{C}$ wurden die Chips mit einer scharfen Klinge herausgeschnitten und Einlässe und Auslässe mit einem Durchmesser von 0,5 mm in beide Enden des Mikrofluidik-Mikroarrays gestanzt. Vor dem Verbinden mit einem PDMS-Mikrofluidchip wurden $25 \times 75 \times 1 \mathrm{~mm}$ Glassubstrate (VWR) in $2 \%$ Hellmanex III-Lösung (Hellma Analytics) 10 Minuten lang beschallt, mit $\mathrm{ddH}_{2} \mathrm{O}$ und Isopropanol gespült und nach dem Föhnen eine Minute lang bei $70^{\circ} \mathrm{C}$ dehydriert. PDMS-Chips und Glassubstrate wurden eine Minute lang plasmabehandelt (Harrick Plasma). Schließlich wurde der PDMS-Mikrofluidchip auf die Oberseite des Glassubstrats gebondet und dadurch die mikrofluidische Einzelzellenarraystruktur versiegelt.

\section{Partikelsuspensionen und Zellkultur}

Für die experimentelle Strömungsvisualisierung wurde eine Probe mit 0,1 Vol.-\% fluoreszierenden 0,2 $\mu \mathrm{m}$ großen Latexteilchen auf Polystyrolbasis (Fluoresbrite ${ }^{\circledR}$ Yellow Green Microspheres, Polysciences) durch Verdünnen der Suspension $\mathrm{dd}_{2} \mathrm{O}$ hergestellt. Die mikrofluidischen Kanäle wurden mit einer sterilfiltrierten Lösung von $2 \mathrm{~g} / \mathrm{L}$ Alginsäurenatriumsalz (Sigma-Aldrich) in $\mathrm{ddH}_{2} \mathrm{O}$ gespült, um ein Anhaften fluoreszierender Partikel an den Wänden der mikrofluidischen Strukturen zu vermeiden. Die menschlichen Choriokarzinom Zelllinien von BeWo b30 wurden in Dulbeccos modifiziertem Eagle's Medium (DMEM mit L-Glutamin und hohem Glucosespiegel; Gibco) und Ham's Nutrient Mixture F12, 10\% fötalem Kälberserum (FCS, PAA) und $1 \%$ Antibiotika (Gibco, Sigma) kultiviert Aldrich). Primäres menschliches ASC wurde in vollständig supplementiertem Endothelzellwachstumsmedium (EGM-2, PromoCell) mit 5\% fötalem Kälberserum bis zur Passage 5-9 aufbewahrt. Alle Zellkulturen wurden in einer angefeuchteten Atmosphäre bei $37^{\circ} \mathrm{C}$ und $5 \% \mathrm{CO}_{2}$ gehalten. Zellkulturmedien, die während der Perfusion des einzelligen Mikroarrays verwendet wurden, wurden mit 0,5\% HEPES (Sigma Aldrich) ergänzt.

\section{Versuchsaufbau}

Der Mikrofluidik-Chip wurde auf eine Heizplatte montiert, die direkt in ein inverses Mikroskop (Olympus, IX71) integriert war, das mit einem Objektiv (Olympus, UPLSAPO100XO) und einer Digitalkamera (Olympus XC10) ausgestattet war, um die Experimente zu überwachen und aufzuzeichnen. Der Mikrofluidik-Chip wurde mit PEEKSchläuchen (Polyetheretherketon) (Außendurchmesser $\varnothing=0,8 \mathrm{~mm}$, Innendurchmesser $\varnothing=0,015 \mathrm{~mm}$, Kinesis) verbunden, die direkt in den Einlass und Auslass des PDMS-Chips integriert wurden. Alle Suspensionen wurden mit 1-mL-Spritzen (Omnifix, Brown) eingeführt, die an eine Spritzenpumpe (neMESYS, Cetoni) angeschlossen waren, um stabile Flussraten bereitzustellen. Vor dem Einbringen von Partikelsuspensionen oder Zellsuspensionen wurden der Mikrofluidchip und die Verbindungsschläuche 30 Minuten lang mit $70 \% \mathrm{EtOH}$ sterilisiert. Zellen wurden in Konzentrationen von $100 \times 10^{3}$ Zellen/mL in den Mikrofluidik-Chip eingeführt. Nach abgeschlossenem

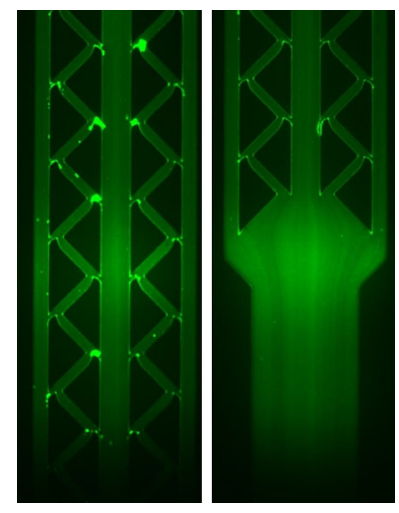

Abb. 2. Mikrofotografien von fluoreszierender Partikelströme im mikrofluidischen Einzelzellen-Microarray bei einer Flussrate von $1 \mathrm{ml} / \mathrm{min}$

Experiment wurde der Mikrofluidik-Chip als Wegwerfprodukt von den Schläuchen getrennt und verworfen.

\section{Experimentelle Strömungsvisualisierung}

Um den Fluss innerhalb der mikrofluidischen Kanalstrukturen und Zellfallen sichtbar zu machen, wurde ein kontinuierlicher Fluss einer Suspension mit fluoreszierenden Partikeln injiziert, um die Stromlinien zu verfolgen. Abbildung 2 zeigt die Fluoreszenzpartikelbahnen bei einer Flussrate von $1 \mathrm{~mL} / \mathrm{min}$. Wie in den Bildern gezeigt, ist in allen Strukturen eine Strömung vorhanden, mit höheren Strömungsgeschwindigkeiten in den Haupttransportkanälen. Innerhalb des Bereichs des Zellfallenhohlraums treten niedrigere Flussraten auf, die das Einfangen einzelner Zellen ermöglichen. Dies wird weiter durch die Ansammlung von Partikeln in den Zellfallen gezeigt.

\section{Einzelzell-Trapping und On-Chip Toxikologie Screening}

Um das mikrofluidische Microarray zu bewerten, wurde die Zelleffizienz mit Suspensionen von BeWo b30-Zellen und Stammzellen (ASC) in zwei getrennten Vorrichtungen getestet. Die Zellen wurden in Konzentrationen von $100 \times 10^{3}$ Zellen $/ \mathrm{mL}$ bei einer Perfusionsrate von $1 \mathrm{~mL} / \mathrm{min}$ ausgesät. Unter diesen Bedingungen wurden Zellen mit einer einzelnen Zelle in jeder Kavität für ungefähr $50 \%$ der gesamten Fallen für beide Zelltypen eingefangen. Nach dem Einbringen und Einfangen von BeWo b30-Zellen und ASC in die Einzelzell-Mikroarrays wurde eine erste Demonstration der On-ChipToxikologie durchgeführt, indem die eingefangenen Zellen Chlorpromazin in einer Konzentration von $1000 \mu \mathrm{g} / \mathrm{mL}$ (Sigma-Aldrich) ausgesetzt wurden. Chlorpromazin (CPZ) ist ein Antipsychotikum, das aufgrund seiner Fähigkeit, Toxizität und oxidativen Stress in verschiedenen Zellen zu induzieren, auch häufig in toxikologischen Modellen verwendet wird [12, 16]. Nach 30-minütiger Inkubation wurde Chlorpromazin mit Zellkulturmedium ausgewaschen und die Zellen wurden mit Ethidiumbromid angefärbt, um zwischen lebensfähigen und nicht lebensfähigen Zellen zu unterscheiden. Bei beiden Zelltypen waren $100 \%$ der eingefangenen Zellen nekrotisch. In Abb. 3 und 4 sind mikroskopische Aufnahmen von eingefangenen BeWoZellen und ASC nach Erfassung und Inkubation mit CPZ mit einem Hellfeldmikroskop und einem Fluoreszenz-mikroskop dargestellt.

\section{Zusammenfassung}

In diesem Artikel wurde ein neuartiges mikrofluidisches EinzelzellenMicroarray zum hydrodynamischen Einfangen und Analysieren von Zellen vorgestellt. Die Herstellung von Formen für die Softlithographie mit $25 \mu \mathrm{m}$ hohen Strukturen mit Details bis zu einer Größe von 

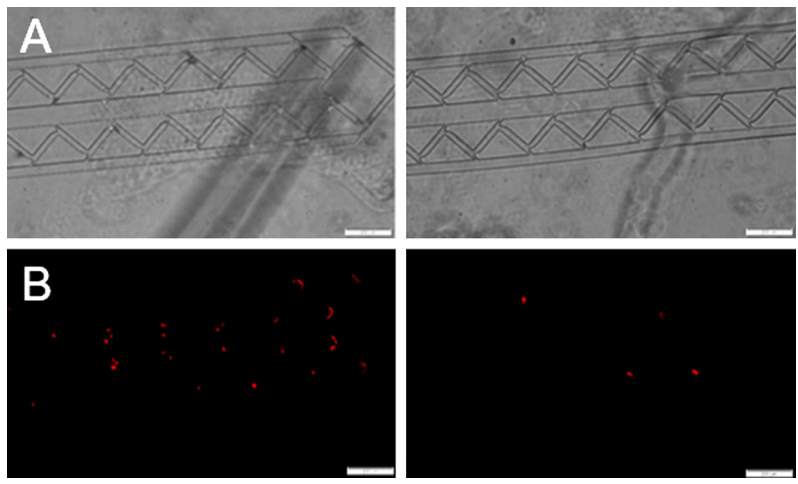

Abb. 3. Mikrophotographien eingefangener BeWo-Einzelzellen nach Chlorpromazin-Behandlung mit einem Hellfeldmikroskop (a) und einem Fluoreszenzmikroskop (b) mit einem roten Farbstoff, der nekrotische Zellen anzeigt. Balkengröß
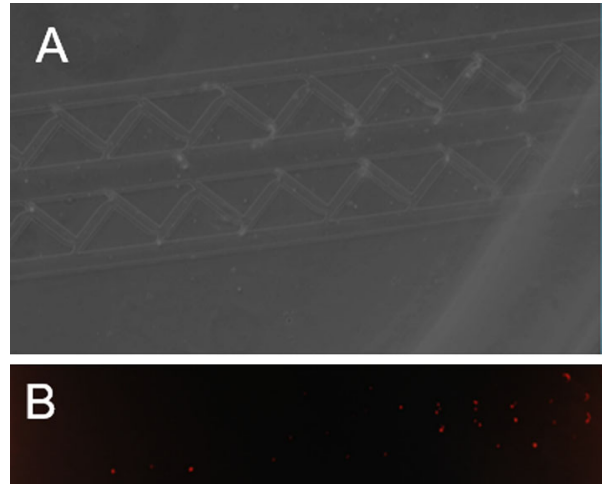

Abb. 4. Mikrophotographien eingefangener ASC-Einzelzellen nach Chlorpromazin-Behandlung mit (a) Hellfeldmikroskop und (b) Fluoreszenz-mikroskop mit rotem Farbstoff als Hinweis auf nekrotische Zellen. 20-fache Vergröß

$8 \mu \mathrm{m}$ wurde mit SU-8-Photolithografie auf Siliziumwafern durchgeführt. Erster Proof-of-Principle-Test zur Erfassung von zwei verschiedenen Zelltypen konnte erfolgreich durchgeführt werden. Zellfangkapazität bis zu $50 \%$ aller Zellen wurden mit den dargestellten Bedingungen erreicht. Parameter wie die Zelldichte und der Perfusionsfluss werden weiter optimiert, um die Zellenerfassungsrate zu erhöhen. Das Einfangen von Zellen war für größere Zellen wie Stammzellen aufgrund des höheren Zelldurchmessers einfacher. Kleinere Zellen wie Epithelzellen könnten sich aufgrund der elastischen Eigenschaften von PDMS durch den Hohlraum quetschen. Nach dem Einfangen und Halten von Zellen in den Hohlräumen wurden Toxizitätstests mit Chlorpromazin mit einem Standard-Farbstoffausschlusstest durchgeführt. Die Integration von Echtzeitsensoren ist jedoch erforderlich, um das Potenzial des entwickelten Einzelzellen-Microarrays voll auszuschöpfen und dynamischere Informationen zur Wechselwirkung von Zellen und Toxine bereitzustellen. Abschließend halten wir das vorgestellte mikrofluidische System für vielversprechend, um die Wechselwirkung zwischen Zellen und Toxinen auf Einzelzellebene zu bewerten.

\section{Danksagung}

Open access funding provided by TU Wien (TUW).

Hinweis des Verlags Der Verlag bleibt in Hinblick auf geografische Zuordnungen und Gebietsbezeichnungen in veröffentlichten Karten und Institutsadressen neutral.

Open Access Dieser Artikel wird unter der Creative Commons Namensnennung 4.0 International Lizenz veröffentlicht, welche die Nutzung, Vervielfältigung, Bearbeitung, Verbreitung und Wiedergabe in jeglichem Medium und Format erlaubt, sofern Sie den/die ursprünglichen Autor(en) und die Quelle ordnungsgemäß nennen, einen Link zur Creative Commons Lizenz beifügen und angeben, ob Änderungen vorgenommen wurden. Die in diesem Artikel enthaltenen Bilder und sonstiges Drittmaterial unterliegen ebenfalls der genannten Creative Commons Lizenz, sofern sich aus der Abbildungslegende nichts anderes ergibt. Sofern das betreffende Material nicht unter der genannten Creative Commons Lizenz steht und die betreffende Handlung nicht nach gesetzlichen Vorschriften erlaubt ist, ist für die oben aufgeführten Weiterverwendungen des Materials die Einwilligung des jeweiligen Rechteinhabers einzuholen. Weitere Details zur Lizenz entnehmen Sie bitte der Lizenzinformation auf http://creativecommons.org/licenses/by/4.0/deed.de.

Literatur

1. Bachmann, B., Spitz, S., Rothbauer, M., Jordan, C., Purtscher, M., Zirath, H., Schuller, P., Eilenberger, C., Ali, S. F., Mühleder, S., Priglinger, E., Harasek, M., Redl, H., Holnthoner, W., Ertl, P. (2018): Biomicrofluidics, 12, 042216

2. Benavente-Babace, A., Gallego-Pérez, D., Hansford, D. J., Arana, S., Pérez-Lorenzo, E., Mujika, M. (2014): Biosens. Bioelectron., 61, 298

3. Di Carlo, D., Aghdam, N., Lee, L. P. (2006): Anal. Chem., 78, 4925

4. Chen, H., Sun, J., Wolvetang, E., Cooper-White, J. (2015): Lab Chip, 15, 1072

5. Dittrich, P. S., Tachikawa, K., Manz, A. (2006): Anal. Chem., 78, 3887.

6. Folch, A., Ayon, A., Hurtado, O., Schmidt, M. A., Toner, M. (1999): J. Biomech. Eng., 121(1), 28-34

7. Gao, D., Jin, F., Zhou, M., Jiang, Y. (2019): Analyst, 144, 766

8. Guo, F., Mao, Z., Chen, Y., Xie, Z., Lata, J. P., Li, P., Ren, L., Liu, J., Yang, J., Dao, M., Suresh, S., Huang, T. J. (2016): Proc. Natl. Acad. Sci. USA, 113, 1522.

9. Hirono, T., Torimitsu, K., Kawana, A., Fukuda, J. (1988): Brain Res., 446, 189.

10. Lagus, T. P., Edd, J. F. (2013): RSC Adv., 3, 20512

11. Lecault, V., White, A. K., Singhal, A., Hansen, C. L. (2012): Curr. Opin. Chem. Biol., $16,381$.

12. López-Muñoz, F., Alamo, C., Cuenca, E., Shen, W. W., Clervoy, P., Rubio, G. (2005): Ann. Clin. Psychiatry, 17(3), 113-135.

13. Love, K. R., Bagh, S., Choi, J., Love, J. C. (2013): Trends Biotechnol., 31, 280.

14. Mitchell, P. (2001): Nat. Biotechnol., 19, 717.

15. Molter, T. W., McQuaide, S. C., Suchorolski, M. T., Strovas, T. J., Burgess, L. W., Meldrum, D. R., Lidstrom, M. E. (2009): Sens. Actuators B, Chem., 135, 678

16. Morgan, K., Martucci, N., Kozlowska, A., Gamal, W., Brzeszczynki, F., Treskes, P., Samuel, K., Hayes, P., Nelson, L., Bagnaninchi, P., Brzeszczynska, J., Plevris, J. (2019): Biomed. Pharmacother., 111, 1408-1416.

17. Reece, A., Xia, B., Jiang, Z., Noren, B., McBride, R., Oakey, J. (2016): Curr. Opin. Biotechnol., 40, 90

18. Rettig, J. R., Folch, A. (2005): Anal. Chem., 77, 5628.

19. Rothbauer, M., Zirath, H., Ertl, P. (2018): Lab Chip, 18(2), 249-270.

20. Sauzade, M., Brouzes, E. (2017): Lab Chip, 17, 2186.

21. Takayama, S., Ostuni, E., LeDuc, P., Naruse, K., Ingber, D. E., Whitesides, G. M. (2001): Nature, 411, 1016.

22. Tice, R. R., Agurell, E., Anderson, D., Burlinson, B., Hartmann, A., Kobayashi, H., Miyamae, Y., Rojas, E., Ryu, J. C., Sasaki, Y. F. (2000): Environ. Mol. Mutagen., 35(3), 206221

23. Valero, A., Merino, F., Wolbers, F., Luttge, R., Vermes, I., Andersson, H., Van Den Berg, A. (2005): Lab Chip, 5, 49.

24. Whitesides, G. M. (2006): Nature, 442,368

25. Wikswo, J. P., Curtis, E. L., Eagleton, Z. E., Evans, B. C., Kole, A., Hofmeister, L. H., Matloff, W. J. (2013): Lab Chip, 13, 3496.

26. Xia, Y., Whitesides, G. M. (1998): Annu. Rev. Mater. Sci., 28, 153 


\section{Autoren}

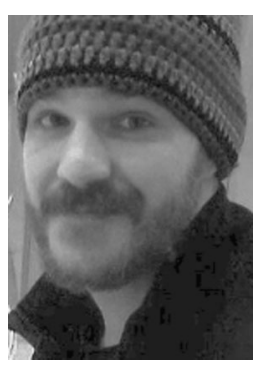

\section{Mario Rothbaue}

provomierte als Dr. nat. techn. in Lebensmittelwissenschaften und Biotechnologie im Jahr 2015 an der Universität für Bodenkultur in Wien (Österreich). Seine Doktorarbeit am AIT Austrian Institute of Technology (Wien, Österreich) beschäftigte sich mit der Entwicklung von Lab-on-a-Chip-Systemen für zellbasierte biomedizinische Anwendungen, bei denen Zellbiologie, Nanobiotechnologie, Biosensoren, Materialwissenschaften und Mikrofabrikations-technologien kombiniert wurden. An der Fakultät für Technische Chemie der Technischen Universität Wien arbeitet er an Lab- und Organ-ona-Chip-Systemen als Alternativen zu Tiermodellen für die Arzneimittelerforschung.

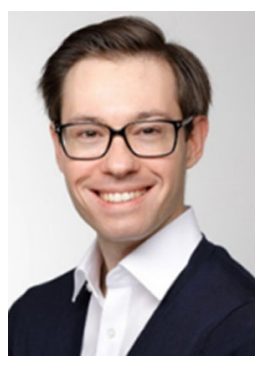

\section{Patrick Schuller}

promovierte 2019 in Elektrotechnik an der Technischen Universität Wien. Seine Doktorarbeit beschäftigte sich mit der Fabrikation von Elektroden auf porösen Membranen und deren Anwendung im biomedizinischen Bereich. Derzeit arbeitet er bei VAMED in der Medizintechnik. Seine Forschungsinteressen sind Mikrosystemtechnik, Biosensoren und zellbasierte Lab-on-a-Chip-Systeme.

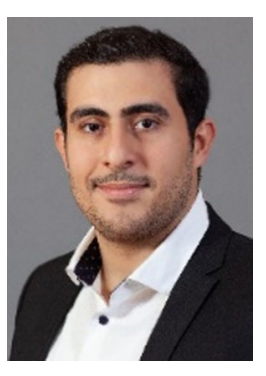

\section{Reza Afkhami}

schloss das Masterstudium als DI in Biomedical Engineering an der Technischen Universität Wien Ende 2019 ab. Im Zuge der Diplomarbeit arbeitete er an einem mikrofluidisches nano-toxikologisches Screening-System mit integrierten Sensoren zum kontinuierlichen Monitoring der Viabilität von Zellen an der Fakultät für Technische Chemie der Technischen Universität Wien.

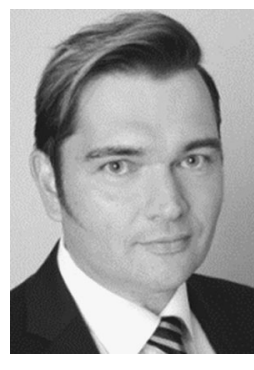

Heinz D. Wanzenboeck

hat einen Abschluss in Technischer Chemie (TU Wien, Österreich). In seiner Doktorarbeit untersuchte er Effekte der oberflächenverstärkten Infrarotspektroskopie. Seine Erfahrung als Post-Doc an der Hokkaido University (Japan) und an der Stanford University (USA) konnte er für seine weitere wissenschaftliche Tätigkeit am Institut für Festkörperelektronik an der Fakultät für Elektrotechnik und Informationstechnologie einbringen. Er ist habilitiert im Fachgebiet "Nanotechnologie" und forscht im Bereich nanotechnologischer Direkt-Schreib-Prozesse und Biomedizinischer Mikrosysteme.

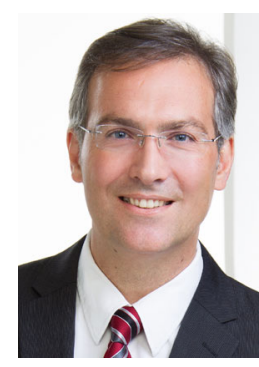

Peter Ertl

hat einen Abschluss in Biotechnologie (BO$\mathrm{KU}$, Österreich), einen Doktortitel in Chemie (Univ. Waterloo, Kanada) und eine Postdoktoranden-ausbildung zum Biophysiker an der University of California in Berkeley (USA). 2005 zog Dr. Ertl nach Österreich, wo er als Senior Scientist in der Abteilung BioSensor Technology am AIT Austrian Institute of Technology tätig war. 2016 wurde er zum Professor für Lab-on-a-Chip-Systeme für biowissenschaftliche Technologien an die Fakultät für Technische Chemie der Technischen Universität Wien berufen. Sein Forschungsschwerpunkt liegt in der Entwicklung von Organ-on-a-Chip- und Chip-in-Organ-Systemen für die biomedizinische Forschung.

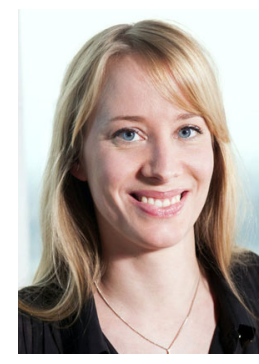

\section{Helene Zirath}

promovierte 2016 in Technischer Chemie an der Technischen Universität Wien und promovierte an der Fakultät für Ingenieurwissenschaften der Universität Lund in Biotechnologie. Ihre Doktorarbeit hat sie an dem Austrian Institute of Technology (AIT, Wien) geführt zum Thema Lab-on-a-Chip-Technologie mit Schwerpunkt Point-of-Care-Diagnostik zur Erkennung von molekularen Markern. Dr. Zirath ist derzeit Postdoc bei der Cell-Chip-Gruppe an der Technischen Universität Wien. Ihre Forschungsinteressen sind Lab-ona-Chip Technologien und Zellchip-Technologien mit Schwerpunkt Nanotoxikologie. 